\title{
Une nouvelle méthode de dosage des acides phénoliques dans les aliments
}

\author{
N Jehl 1,2, D Debroas 2, G Blanchart 2 \\ IINRA, Station de recherches cunicoles, 31320 Castanet Tolosan ; INRA-ENSAIA, \\ laboratoire de zootechnie, 54505 Vandoeuvre, France
}

\begin{abstract}
Les acides phénoliques non polymérisés permettent de relier les hemicelluloses à la lignine. L'élimination de ces composés pourrait augmenter la dégradation de la paroi pectocellulosique (Jung et Sahlu, 1986, J Sci Food Agric, 37, 659). II a été montré in vitro que ces monomères de lignine possédaient une activité inhibitrice sur la croissance des bactéries cellulolytiques (Chesson et al, 1986, Appl Environ Microbiol, 44, 597). Les acides phénoliques pourraient par conséquent jouer un rôle important dans la dégradation des parois végétales. Nous exposerons dans cette étude une nouvelle méthode pour doser ces composés dans les parois.
\end{abstract}

Préparation des parois. $1 \mathrm{~g}$ d'aliment en suspension dans $20 \mathrm{ml}$ d'eau est placé $1 \mathrm{~h}$ à $90^{\circ} \mathrm{C}$ puis $3 \mathrm{~h}$ à $60^{\circ} \mathrm{C} .80 \mathrm{ml}$ d'éthanol sont rajoutés à froid. Le mélange est incubé pendant $12 \mathrm{~h}$ à $4^{\circ} \mathrm{C}$. Le résidu insoluble dans l'éthanol (RIE) est filtré sur creuset puis séché à l'étuve à $80^{\circ} \mathrm{C}$. Cette méthode permet d'éviter une solubilisation des monomères de lignine.

Extraction des acides phénoliques. $500 \mathrm{mg}$ de parois sont mélangés à $5 \mathrm{ml}$ de $\mathrm{NaOH} 1 \mathrm{M}$ contenant un étalon interne (acide $3,4,5$ trimethoxycinamique) à $1 \mathrm{mg} / \mathrm{l}$. L'hydrolyse est réalisée à $39^{\circ} \mathrm{C}$ pendant $24 \mathrm{~h}$ en présence d'azote. Le mélange est filtré sur $8 \mu \mathrm{m}$.

Dérivation. Une méthode, décrite récemment par Husek (1991, J Chromatogr, 547, 289), basée sur l'utilisation du (chloro)alkyl chloroformate a été utilisée. L'étude de tous les paramètres de dérivation a montré que celle-ci est optimale pour un $\mathrm{pH}$ neutre. Par conséquent le produit de l'extraction est neutralisé par $\mathrm{HCl} .50 \mu \mathrm{l}$ de l'échantillon à analyser sont mélangés à $10 \mu \mathrm{l}$ d'étalon interne (acide 3, 3, 4 diméthoxyphenyl propionique à 1 $\mathrm{mg} / \mathrm{ml}$ dans un tampon phosphate $0,01 \mathrm{M}$ ) puis à $40 \mu \mathrm{l}$ d'une solution éthanol-pyridine $(4: 1 \mathrm{~V} / \mathrm{v})$. L'addition de $5 \mu \mathrm{l}$ d'éthyle chloroformate (ECF) permet de dériver les acides phénoliques qui sont alors extraits par une solution de chloroforme contenant $1 \%$ d'ECF.

Analyse. La séparation des acides phénoliques est réalisée sur une colonne capillaire OV 1701 greffée (25 $\mathrm{m}$, diamètre interne 0,32 $\mathrm{mm}$ Spiral, Dijon) par une programmation de température de $155^{\circ} \mathrm{C}$ à $270^{\circ} \mathrm{C}$ à $10^{\circ} \mathrm{C} / \mathrm{min}$.

Cette méthode permet d'analyser les principaux acides phénoliques présents sous forme de monomères dans les parois végétales (acide trans cinnamique, acide hydroxybenzoïque, acide vanilique, acide p-coumarique et acide férulique). Elle a été testée sur des aliments différents et a permis d'obtenir des résultats reproductibles. L'avantage de cette méthode réside dans la rapidité de la réaction de dérivation (moins de 1 minute) et le faible coût des réactifs.

Teneurs en acide p-coumarique et férulique ( $\mathrm{mg} / \mathrm{g}$ de MS de RIE), moyennes et écarts types sur quatre répétitions

\begin{tabular}{lccc} 
& $\mathrm{n}$ & acide $\mathrm{p}$-coumarique & acide férulique \\
\hline Paille & 4 & \multicolumn{2}{c}{ (mg/g de MS de RIE) } \\
Pulpe de betteraves & 4 & $3,28 \pm 0,01$ & $2,40 \pm 0,10$ \\
Son & 4 & traces & $9,05 \pm 0,31$ \\
\hline
\end{tabular}

